\title{
Polymorphism of flax pathogens assessed using deep sequencing
}

Novakovskiy R.O. ${ }^{1}$, Krasnov G.S. ${ }^{1}$, Pushkova E.N. ${ }^{1}$, Kudryavtseva L.P. ${ }^{2}$,

Rozhmina T.A. ${ }^{1,2}$, Melnikova N.V. ${ }^{1}$, Dmitriev A.A. ${ }^{1 *}$

${ }^{1}$ Engelhardt Institute of Molecular Biology, RAS, Moscow, Russia

${ }^{2}$ Federal Research Center for Bast Fiber Crops, Torzhok, Russia

*e-mail: Alex_245@mail.ru

Pathogens decrease the crop yield and reduce the quality of products obtained from flax (Linum usitatissimum L.). The knowledge on the genetic diversity of flax pathogens is necessary for the development of molecular marker system for fungus identification and subsequent application of proper defense actions. In the present work, the polymorphism of fungal pathogens of flax was assessed using deep sequencing. Twenty-four strains of Fusarium oxysporum, 8 strains of Fusarium moniliforme, 5 strains of Fusarium solani, 5 strains of Fusarium culmorum, 4 strains of Fusarium gibbosum, 3 strains of Fusarium sporotrichiella, 3 strains of Fusarium avenaceum, 2 strains of Fusarium semitectum, 20 strains of Colletotrichum lini, 9 strains of Melampsora lini, 9 strains of Aureobasidium pullulans, and 8 strains of Septoria linicola were obtained from Institute for Flax (Torzhok, Russia). Internal transcribed spacer (ITS) region of the rRNA genes and regions of genes encoding translation elongation factor 1-alpha (tef1), beta-tubulin (tub2), and RNA polymerase II subunits (RPB1 and RPB2) were chosen for assessment of genetic diversity of flax fungal pathogens. For DNA library preparation, the method proposed by Illumina for $16 \mathrm{~S}$ metagenomic sequencing library with some modifications was applied. Primers with overhang adapters were used at the first stage of DNA library preparation followed by the second stage of amplification using Nextera XT index primers. DNA libraries were obtained for 100 fungal pathogen strains and sequenced on MiSeq Illumina with 300+300 nucleotide read length. About 4000 reads were generated for each sample. The pipeline was developed for the analysis of the sequencing data. The results of our work allow to characterize the genetic diversity of flax fungal pathogens and to determine the DNA sequences that are the most suitable for identification of fungus that cause $L$. usitatissimum diseases using molecular markers.

Acknowledgements: This study was supported by the Russian Science Foundation. This work was financially supported by the Russian President Grant MK-5828.2018.4. 